\title{
Tradición, Sentido y Formación en el Desarrollo de las Albañilas en Latinoamérica
}

\author{
Tradition, Meaning and Training in the Development of Female Bricklayers in \\ Latin America
}

\section{Tradição, Significado e Treinamento no Desenvolvimento de Pedreiros Mulheres na América Latina}

\author{
Wendy Margarita Montes Ponce \\ Doctora en Arquitectura, Universidad Autónoma Benito Juárez de Oaxaca, México. \\ wmontes.cat@uabjo.mx \\ (iD) https://orcid.org/0000-0003-1962-4676 \\ Otniel Josafat López Altamirano \\ Doctor en Design, Universidad Autónoma Benito Juárez de Oaxaca, México. \\ otniel_digital@hotmail.com \\ (iD) https://orcid.org/0000-0001-7593-9715
}

Recibido: mayo 31 de 2020

Aceptado: junio 8 de 2020

Publicado: junio 15 de 2020

\section{RESUMEN}

La participación de las Albañilas en la construcción se ha incrementado en los últimos años. La formación en el oficio representa desventajas que tienen origen, tanto en lo biológico como en el género. El presente artículo tiene la finalidad de exponer las condiciones bajo las cuales se desarrollan las Albañilas en Latinoamérica, demostrando que el reconocimiento laboral ha experimentado ensambles políticos, empresariales y sociales, hasta lograr su formalización de aprendizaje académico, potenciando sus saberes, habilidades y economía familiar. Los avances han rendido frutos positivos. Sin embargo, se requieren políticas públicas permanentes que fortalezcan los programas académicos, implicando los ambientes urbanos y rurales.

Palabras clave: Construcción, Albañila, educación, Latinoamérica, género.

\section{ABSTRACT}

The participation of Female Bricklayers in construction has increased in recent years. Training in the trade represents disadvantages that originate, both biologically and gender. The purpose of this article is to expose the conditions under which Female Bricklayers develop in Latin America, demonstrating that labor recognition has undergone political, business and social assemblies, until achieving their formalization of academic learning, enhancing their knowledge, skills and family economy. Progress has paid off positively. However, permanent public policies are required to strengthen academic programs, involving urban and rural environments.

Keywords: Construction, Female Bricklayers, education, Latin America, gender. 


\section{RESUMO}

A participação de Pedreiros Mulheres na construção aumentou nos últimos anos. O treinamento no comércio representa desvantagens originárias, tanto biológicas quanto de gênero. O objetivo deste artigo é expor as condições sob as quais os Pedreiros Mulheres se desenvolvem na América Latina, demonstrando que o reconhecimento do trabalho passou por assembléias políticas, empresariais e sociais, até alcançar a formalização do aprendizado acadêmico, aprimorando seus conhecimentos, habilidades e economia familiar. O progresso valeu a pena positivamente. No entanto, políticas públicas permanentes são necessárias para fortalecer os programas acadêmicos, envolvendo ambientes urbanos e rurais.

Palavras-chave: Construção, Pedreiros Mulheres, educação, América Latina, gênero.

\section{INTRODUCCIÓN}

En la historia de la construcción arquitectónica, la participación de la mano de obra ha sido predominantemente masculina. Sin embargo, los tiempos contemporáneos han reintegrado al oficio la fuerza laboral de las mujeres, dentro del quehacer de la Albañilería. Desde esta observación, creemos meritorio desarrollar estudios que expongan las circunstancias y los factores que experimentan las Albañilas. Por ello, el presente artículo expone los resultados de una investigación que poca atención ha tenido en el universo disciplinar de la Arquitectura.

El objetivo de este trabajo documental está centrado en mostrar la participación de las Albañilas en Latinoamérica-concretamente en México, Argentina, Bolivia, Brasil, Colombia, Ecuador, Paraguay, República Dominicana y Uruguay-, reconociendo, en este problema contemporáneo, variables de estudio que han impedido de manera considerable la incorporación de las mujeres a esta actividad económica, por tratarse de condiciones y causas predominantemente culturales (Figura 1). Para darle alcance al objetivo, se pensó en accionar una metodología que, a través del análisis de Casos de Estudio, demostraran condiciones espacio-temporales de las actividades que ellas desempeñan, observando los factores y circunstancias implícitas en su formación laboral. La consulta de documentos demostró intereses políticos y sociales que han impulsado programas académicos con certificación escolarizada, que les brinda mejores oportunidades de empleo.

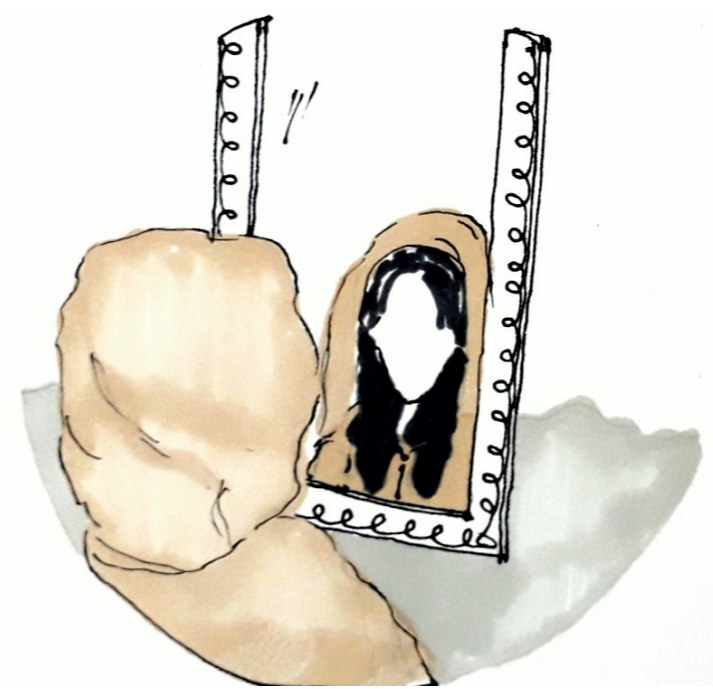

Figura 1. La Conciencia de ser mujer Albañil Fuente: Flor Ríos Ventura

El planteamiento de estudio de manera inicial condujo a preguntas tales como: ¿Qué significa ser mujer Albañil? ¿Qué función tiene en las tareas de la construcción arquitectónica? ¿Por qué no se les reconoce de manera más amplia? La hipótesis expresa que la exposición de las mujeres Albañiles frente a los ámbitos laboral, social y político, posibilitará su incorporación en esta actividad económica, con mayores oportunidades de crecimiento organizacional, salarial y profesional, fracturando las condiciones y causas culturales actuales. De modo que, para los fines ya explícitos, el artículo se constituye de tres apartados que exploran la tradición de ser Albañil, el sentido de ser mujer Albañil y la formación académica de las Albañilas. 


\section{LA TRADICIÓN DE SER ALBAÑIL}

En el oficio de la Albañilería se han localizado registros sobre la intervención femenina desde tiempos lejanos. Casos concretos se exponen en el estudio que hizo María Teresa López Beltrán, sobre el trabajo de las mujeres en la España medieval, quien explica que, en las obras de construcción durante el siglo XIV, algunas mujeres practicaron labores de auxiliar de Albañiles, obteniendo salarios regulares establecidos por las Cortes, y cita "(...) En la ciudad de Burgos, las mujeres trabajaban en las obras públicas haciendo la mezcla o transportando el agua..." (López, 2010, p. 48), mencionando, también, que existieron obreras en la Catedral de Toledo durante los inicios del siglo $X V$. Lo anterior da cuenta de que este oficio no era de exclusividad masculina, pero que, sin embargo, si lo era predominantemente varonil.

El concepto "Albañil" se define como: "Persona que se dedica profesionalmente a la Albañilería" (RAE, 2019), por lo que se infiere que el oficio puede estar ejercido tanto por hombres, como por mujeres.

Es de llamar la atención que los manuales de Albañilería más antiguos, como el que fuera publicado por la Biblioteca Enciclopédica Popular llustrada en 1879, en la ciudad de Madrid, escrito por el Arquitecto D. Ricardo Marcos y Bausá, no puntualicen una definición sobre el término "Albañil", o cuando menos qué facultades debía poseer. La introducción del documento señalado, sin embargo, justifica su creación argumentando:

Con el objetivo de establecer riguroso orden y método en la exposición de la doctrina del libro, haciéndolo todo claro y comprensible (...) dividido en tres partes, que juntas componen todo lo que deben saber las personas que a la Albañilería se dedican (...) para lograr la claridad apetecida y vulgarizar el conocimiento de tan útil arte mecánico, contribuyendo al mismo tiempo a que su aprendizaje sea algún tanto razonado y no tan puramente rutinario como en el día. (Marcos y Bausá, 1879, p. 7).

En efecto, la edición de documentos como este, hace posible estimar que el oficio de Albañil tuvo un alto reconocimiento gremial, pero que, además, existió un profundo interés por instruir a los Albañiles fuera de los aprendizajes informales o empíricos.
Transcurrido el tiempoy gracias al interés cimentado en el aprendizaje escolarizado de los Albañiles, se institucionalizó en algunos países latinoamericanos el "Proyecto Multinacional de Desarrollo Educativo Integrado en Zonas Desfavorables"; iniciativa que se constituyó entre los países de Argentina, Bolivia, Paraguay y Uruguay. Impulsado por la Organización de los Estados Americanos - OEA, y, promovido a través de la Secretaría del Área de Frontera "Jáchal", del Ministerio de Educación y Justicia de la República de Argentina, 1987, puso en marcha el Curso de Albañilería Práctica, considerando la Guía Teórica del proyecto PEM-OEA/760, en la localidad de Colanguil - Departamento de Iglesia, Provincia de San Juan.

Una revisión al proyecto citado, mostró en su estructura definiciones generales de Albañilería, destacando cada uno de los trabajos que se debían aprender. Especifica dentro de sus páginas, a modo de listado, materiales, artefactos, herramientas y utensilios que se requerían dominar, indicando las instrucciones textuales y dibujos descriptivos, relacionados con el trabajo de la Albañilería, demostrando que el oficio de la Albañilería implicaba tareas constructivas de gran esfuerzo físico (Figura 2).

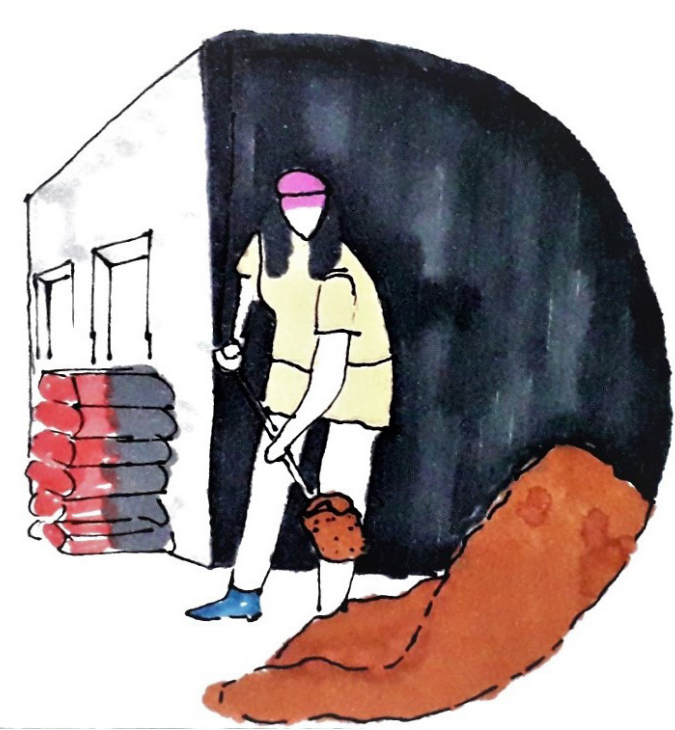

Figura 2. Trabajo que requiere de Fuerza Física Fuente: Flor Ríos Ventura

En México, instituciones de alto prestigio como la Universidad Nacional Autónoma de México (UNAM), han promovido la profesionalización 
de obreros en la construcción, con el objetivo de certificar sus saberes adquiridos inicialmente de manera experimental. Fue en 2014 -con la creación de la Escuela Mexicana de la Construcción (EMC), acreditada por la Secretaría de Educación Pública y el Consejo Nacional de Normalización y Certificación de Competencias Laborales, de manera conjunta con la Facultad de Arquitectura (FA) de la UNAM, desde la División de Educación Continua y Actualización Docente-, que el aprendizaje de los Albañiles se emprendido de manera áulica y periódica.

La capacitación de los Albañiles, a través de esta institución, tiene por objetivo no sólo perfeccionar las técnicas de construcción, sino también posibilitar su certificación: (...) concluidas las clases, se extienden reconocimientos por cada unidad temática acreditada, a través de planos arquitectónicos, instalaciones hidráulicas, instalaciones sanitarias, instalaciones eléctricas, Albañilería, acabados, y por planos estructurales. Reconocido programa escolarizado para la superación de los trabajadores de la construcción (Universidad Nacional Autónoma de México, 2016).

La formación de los Albañiles en Latinoamérica aún sigue siendo un aprendizaje empírico adquirido en sitio. El ingreso del Albañil como aprendiz arranca desde temprana edad, y la enseñanza que recibe se acompaña de maltrato y bajos salarios. Ya sea que se trate de una inducción familiar, es decir, que el padre ofrezca en mano de obra al hijo, o que el infante llegue de manera voluntaria a la construcción, así, desde su incorporación, el sujeto recibe una categoría y una promesa de crecimiento laboral que puede alcanzar hasta el reconocimiento de maestro de obra. Luego, entonces:

Los ayudantes de Albañiles no requieren casi ninguna preparación, solamente el "aguante" para cargar materiales, hacer excavaciones y revolver la mezcla, entre otros, de ahí que es el primer paso para aprender el oficio; a algunos ayudantes les nombran "primera cuchara" cuando ya tienen algún grado de experiencia en algunas labores propias de un Albañil en forma; de cualquier manera, el sueldo entre un ayudante y un "media cuchara", por lo general, es el mismo (González, 2017).

No obstante, el orden del aprendizaje y el tiempo de permanencia en cada puesto, ayudante de Albañil, media cuchara, peón, chalan o Albañil, al no depender de una formación escolarizada, se convierten en factores cuestionables e inciertos. De manera que, para cuando el reconocimiento de aquel ayudante llega a la calificación de Albañil, este sujeto ha adquirido adiestramientos de diversos tipos, como lo son de carácter laboral, de autoridad y de arbitrariedad. Y, todo ello, culturalmente se ha nutrido y justificado con base en la rudeza del oficio y las capacidades del sujeto que lo ejerce.

\section{LA DECISIÓN DE SER ALBAÑIL SIENDO MUJER}

La participación de la mujer en el oficio de la Albañilería, en las últimas décadas, se ha incrementado. Evidencia de ello son los datos estadísticos que se han publicado en los países latinoamericanos. En México, a través del Instituto Nacional de Estadística y Geografía, se reportó en 2013 que "(...) los Albañiles representan el 4.8\% de la población ocupada en México; de esta el 99.6\% de ellos son hombres, mientras que el $0.4 \%$ son mujeres" (Universidad Veracruzana, 2017). Ahora bien, en Bolivia, los datos extraídos del diagnóstico socioeconómico de 2012, demuestran que se encuestaron a 418 mujeres Albañiles localizadas en Ciudad la Paz, trabajadoras en diversas empresas públicas y privadas (Red del Habitat, 2012a).

Su determinación, como trabajadora de la construcción, obedece a circunstancias y factores relacionados a la superación personal y familiar. Existen evidencias en la que el esposo o algún pariente impulsa a la mujer a solicitar empleo en la obra, donde este ejerce la Albañilería, advirtiéndole de ante mano que las condiciones laborales y personales serán diferentes a las que tienen derecho los hombres Albañiles. Como en el caso de José María: "Yo si tengo trabajo con mi mujer por aquí. Hace un año no más que trabajé aquí con mi mujer, ella trabajaba en la construcción conmigo. Ella ganaba 600,500 , yo ganaba 1200 , casi tres meses demorábamos (...)" (Mauro, 1986, p. 69). De manera que la toma de decisión desde el origen se encuentra condicionada por factores de desigualdad provenientes de lo biológico, el género, los saberes, la jerarquía, los derechos y el salario.

Las tareas que desempeña la mujer Albañil al incorporarse a la obra están relacionadas con el acarreo de materiales, cavar zanjas, palear la mezcla, recoger escombros, limpiar las áreas, 
remojar tabiques y llenar los tambos con agua. Actividades que están acompañadas de maltrato psicológico, verbal, físico y de hostigamiento sexual. Así, la obrera se enfrenta a necesidades propias de su género. El cambio de ropa, que resulta ser un evento necesario para los Albañiles, representa en ella un problema de integridad; más aún cuando se trata de la necesidad de aseo personal e íntimo, pues, por lo regular, no se cuenta con un sanitario, teniendo que resolver su necesidad con los vecinos de la zona.

Desde luego que existen otras circunstancias de integración laboral para las mujeres Albañiles. Historias que están circunscritas en los programas de capacitación, cuyo objetivo es brindar un servicio de enseñanza a mujeres que buscan aprender el trabajo de la Albañilería para resolver labores de construcción dentro de sus hogares, y, con ello, fomentar el ahorro. No obstante, algunas con el tiempo llegan a contratarse de manera formal en alguna empresa o con algún constructor independiente.

La contratación de las mujeres Albañiles no resulta sencilla, principalmente por las desventajas que se le adhieren al género. Se trata de una calificación que emana de variables comparativas con base en capacidades específicas, tales como la fuerza, la resistencia, el rendimiento, la constancia (ver Figura 3). Es decir: "La mujer Albañil muestra ser una obrera con conocimientos y expectativas limitadas en el rubro de la construcción (...) Es una obrera que, a pesar de los años en el rubro de la construcción, se mantiene en la categoría de ayudante." (Red del Hábitat, 2012a, p.43).

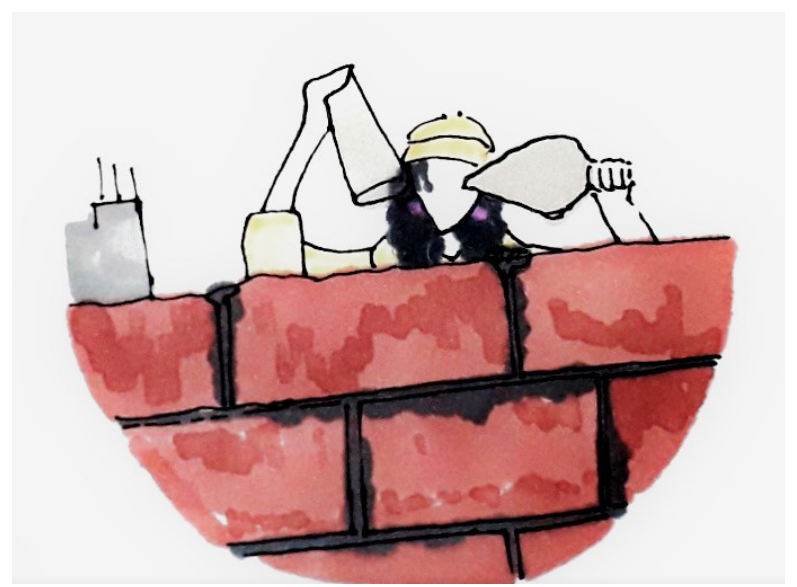

Figura 3. Una Labor que se Evalúa por Rendimiento Fuente: Flor Ríos Ventura
Así, los empleadores han encontrado ventajas en la mano de obra femenina. Motivados por la reducción de costos en la inversión del recurso humano, y sin garantías de pago por concepto de prestaciones de ley, estas acciones les han generado mayores ganancias. De manera que: "(...) las mujeres, quienes son consideradas como fuerza de trabajo secundario, que pueden ser contratadas sin derechos laborales, con bajos salarios y sin posibilidades de sindicalización (...)". (Red de Hábitat, 2012b, p. 11).

Las estadísticas presentan una constante: la edad promedio más temprana en las mujeres Albañiles se registra a los 15 años y alcanza los 60 años en la edad de mayor madurez. De ahí, que se les califique como aprendices, pues se trata de empleadas de la construcción con historias laborales remotamente compatibles:

La baja calificación de su mano de obra se debe principalmente a que ingresaron al rubro a una edad madura y después de haber transitado por varios empleos (trabajadora del hogar, comercio, etc.), (...) obligadas por las circunstancias de generar ingresos (...) (Red del Hábitat, 2012b, p. 44).

En efecto, el aprendizaje de las mujeres Albañiles resulta ser rudo, en comparación al de los aprendices masculinos. Los maestros de obra al asignarles tareas, les encomiendan trabajos que implican palear, acarrear, mezclar, limpiar; empero, las trabajadoras más hábiles aprenden otras tareas del oficio, llegando en algunos casos a ocupar cargos de maestro contratista; aunque en otros tantos casos no aspiran a poseer una categoría tan alta, por no asumir obligaciones mayores, tal como lo narra en septiembre de 2010, Sonia Choquetarqui, Maestra Contratista:

Ser maestra contratista no es chiste ya que tienes que asumir muchas responsabilidades porque manejas dinero, ya hay contratos de por medio, tienes que hacerte respetar con tus obreros y obreras, pero también tienes que conocer bien el oficio de Albañil, saber obra gruesa, obra fina, leer planos, etc. (Red del Hábitat, 2012b, p. 46). 
Por lo anterior, conviene distinguir que la inserción de la mujer al campo laboral de la Albañilería se debe a la precarización del empleo. La disminución de los miembros de la familia, el fenómeno migratorio y la relajación del compromiso en las uniones de pareja, conllevan a la obligatoriedad laboral de la mujer (Arias, 2016, p. 199), involucrándose en áreas de trabajo que antes ocupaban de manera exclusiva los varones.

Ciertamente, la mujer Albañil, circunscrita en desigualdades y desprotección laboral, justifica sus circunstancias a través de las condiciones informales de su empleo. Inclusive, experimenta sensaciones de agradecimiento al ser beneficiaria de ropa, aunque esta no sea de su utilidad por no corresponder a su estilo o talla:

(...) afirman haber sido provistas de ropa de trabajo (...) una de cada dos mujeres no usa las ropas de trabajo como: chalecos, botas, overol hasta casco, más sí guantes de trabajo (...) indican que les es incómodo utilizar esos implementos, especialmente casco, botas y overol... [Pues] las hace ver como varones no como mujeres (...). (Red del Habitat, 2012a, p. 40).

Esta sensación no es la única que las Albañilas abrigan. El miedo ante el desconocimiento del trabajo, el nerviosismo por el trato que reciben, el cansancio por la rudeza que implican las tareas, la culpabilidad por no dedicar más tiempo a los hijos y al hogar, y la satisfacción por el autorreconocimiento de sus habilidades y aptitudes, son otros tantos de los sentimientos que se disipan o acrecientan durante las jornadas laborales.

De manera que no es difícil imaginar el contexto de trabajo para las mujeres Albañiles, acompañado de actividades propias de un ama de casa, el desempeño materno y de esposa; incluso, en el cometido de cuidadora con sus adultos mayores, lo cual reduce singularmente el tiempo para las oportunidades de capacitación en el oficio de la Albañilería u otro.

\section{LA FORMACIÓN ESCOLARIZADA DE LA MUJER ALBAÑIL}

En el Manual de Albañilería para obras de saneamiento básico, se especifica que "albañil" "Es una persona entrenada, hombre o mujer, que trabaja en la construcción de letrinas, tanques de agua, casas, edificios, utilizando materiales como piedra, arena, grava, ladrillo, cal, yeso, cemento, madera, etc." (Zambrana y Saavedra, 1992); infiriéndose que la mujer dedicada a este oficio, en 1992, era identificada bajo el vocablo masculino de Albañil. Por el contrario, en 2017, durante el Tercer Foro Regional de Equidad y género "Diferentes miradas", organizado por la Universidad Veracruzana, México, se empleó el neologismo de Albañilas. La ponencia "La participación de las mujeres en la mano de la industria de la construcción", está centrada en demostrar a través de datos estadísticos y sociales, la inclusión de mano de obra de las mujeres en la construcción.

De manera que, la visibilidad de las Albañilas no es un asunto nuevo; su reconocimiento en los últimos años ha sido tan notable, que algunos gobiernos latinoamericanos han formalizado el aprendizaje del oficio a través de programas escolares, diseñando cursos, talleres y academias. Se trata de esfuerzos que conjugan la participación de instituciones del estado, empresas privadas, institutos académicos $y$ fundaciones no gubernamentales:

La construcción ha demostrado ser en la actualidad uno de los rubros que mayor empleo genera en Bolivia y requiere mayor demanda de mano obra... Paralelamente, esta situación ha propiciado que también instituciones del Estado, la empresa e instituciones privadas, estén interesadas por potenciar este sector promoviendo programas de capacitación, financiando y viabilizando proyectos dirigidos al sector especialmente obrero de la construcción. Uno de ellos son los programas de capacitación que, en algunos casos ya existían, y otros son de reciente creación. Se trata de programas de formación de mano de obra para su inserción en el mercado laboral y otras para el mejoramiento de las competencias laborales (...). (Red del Hábitat, 2012a, p. 42).

La investigación de Jorge Malpica, presentada en la Universidad Politécnica de Valencia, apunta: "La Cámara Mejicana de la Industria de la Construcción (CMIC) y el Instituto Jalisciense de las Mujeres (IJM) firmaron un convenio para la capacitación en oficios tales como Albañilería, electricidad, fontanería, carpintería y manejo de maquinaria pesada..." (Malpica, J. 2018, p. 71). Mientras que, en el Tercer Foro Regional de Equidad y Género, se mencionó el ejercicio de capacitación organizado 
por la Delegación de Tlalpan, en Ciudad de México, evento certificado por la empresa CEMEX: "Más de 40 mujeres que habitan en colonias aledañas al centro histórico de Tlalpan decidieron romper con la tradición y se inscribieron a un curso de Albañilería y autoconstrucción" (Universidad Veracruzana, 2017).

En Argentina, se promovió un programa escolarizado en el que se involucraron instituciones como la Cámara Argentina de la Construcción; la Unión de la Construcción de la República Argentina; el Instituto de Estadísticas y Registro de la Industria de la Construcción; el Ministerio de Trabajo, Empleo y Seguridad Social y la Formación Continua, iniciativa, para la cual se formuló un organismo de supervisión:

El Ministerio de Trabajo, Empleo y Seguridad Social de la Nación, en el marco de sus políticas activas para la promoción del empleo y la formación profesional, implementa estrategias de apoyo a la competitividad económica y la equidad social.

En este marco y con el objeto de asegurar la calidad del empleo y el desarrollo de las competencias de la población trabajadora, afines a la demanda de calificación laboral actualizada y potencial del mercado laboral, desarrolla desde la Unidad de Evaluación Monitoreo y Asistencia Técnica UEMAT (...). (Sistema Nacional de Formación Continua y Certificación de Competencias, 2017).

Ciertamente, se trata de programas que pretenden desarrollar una capacitación teórica y práctica, desde la cual las mujeres se inserten en el oficio de la construcción, con mayores habilidades, lo que representa para el empleador encontrar en este recurso humano una fuerza laboral más completa.

De acuerdo con las fuentes consultadas, en 2014, el "Programa Mujeres en la construcción", impulsado por el Gobierno del Estado de México, coordinado por la Cámara Mexicana de la Industria de la Construcción y el Consejo Estatal de la Mujer y Bienestar Social (CEMYBS), la capacitación que recibieron las aprendices inscritas incluía la enseñanza detareas relacionadasconlaelectricidad, el recubrimiento de muros, la instalación de pisos y azulejos, y plomería (ver Figura 4); proceso de aprendizaje que incluyó apoyos en especie, como herramientas y equipo. No obstante, a pesar de que el programa actualmente se encuentra suspendido, el reporte de capacitación registró un total de 40 mil mujeres beneficiadas. (Sánchez, 2014).

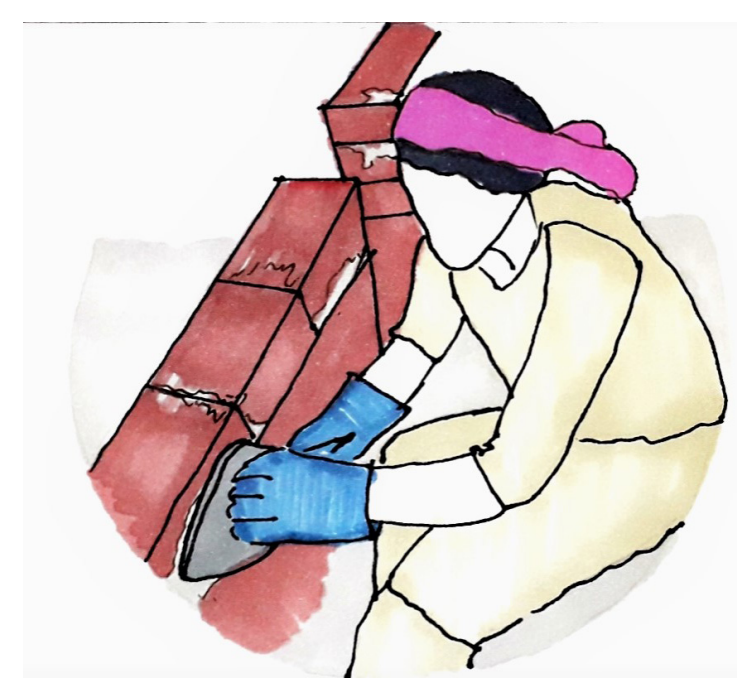

Figura 4. Capacitación de Albañilas con Apoyo Institucional Fuente: Flor Ríos Ventura.

Bolivia, representa uno de los países que ha promovido en los últimos diez años la formación de Albañilas, a las que identifica como mujeres constructoras. La Red Hábitat, a través del Proyecto "Mypes, Mujeres Constructoras del Hábitat", y con el apoyo del Fondo de Emancipación CONEXIÓN, desarrolló una investigación diagnóstica que reveló:

(...) la realidad de las mujeres que están dedicadas al rubro de la construcción. Los resultados (...) pretenden ser un valioso instrumento para orientar el diseño de políticas públicas que impulsen (...) los derechos laborales de las mujeres constructoras (...) (...) tomando en cuenta las potencialidades que tienen para especializarse en rubros de acabados, pintura, instalaciones sanitarias, y otros... para dejar de ser solo las ayudantes de la construcción (...). (Red del Hábitat, 2012a).

Con base en ese estudio, el perfil formativo de la Albañila posee fortalezas que destacan la limpieza, el orden, el cuidado, el entusiasmo, la administración y la responsabilidad laboral. De ahí, que estos atributos otorgan áreas de oportunidad, pues las empresas y maestros de obra observan en ellos una valoración distinta del trabajo de la empleada de la construcción. Por otra parte, durante ese estudio 
diagnóstico se detectaron factores de amenaza, vinculados con la inseguridad emocional y física de la mujer dentro de los ambientes de obra, y otros factores ocasionados por el acoso laboral y sexual; agregándose el factor de inestabilidad económica del ramo constructivo y las fluctuaciones en el mercado de trabajo.

Las Albañilas han reconocido una necesidad de capacitación en: "Obra fina, obra gruesa, empedrado, adoquinado, cordones de acera, cálculo de materiales y presupuesto, lectura de planos, conexión eléctrica, tabiquear, conocer de contratos, manejo de maquinaria pesada y encofrados; leer, escribir y multiplicar; almacenaría". (Red del Hábitat, 2012a, p. 50). Por ello, la Red Hábitat gestionó en los siguientes meses un financiamiento; para diseñar y emprender cursos básicos de construcción, certificando a más de dos centenas de mujeres:

El proyecto fue diseñado para mejorar la preparación técnica de las mujeres dedicadas a la construcción. Se realizaron 8 cursos básicos de construcción con la certificación del Centro de Capacitación y Adiestramiento Municipal (CCAM) del GAMLP [Gobierno Autónomo Municipal de La Paz], capacitando a las mujeres en obra gruesa, obra fina y acabados, pero apostando a que se especialicen en instalaciones de servicios y pintura, entendiendo que estos son los rubros que las mujeres podrían cubrir con mejores competencias en el mercado. Se capacitó a 237 mujeres, de las cuales más del 65\% fueron obreras de diferentes unidades operacionales del GAMLP (Red del Hábitat, 2012b, p. 50).

Una segunda etapa de trabajo, planeada por esta misma red, se enfocó en una investigación relacionada con el diseño de cursos de capacitación, desarrollando temáticas vinculadas con los derechos laborales de las Albañilas, las políticas públicas, la potencialización de las capacidades y competencias en el ámbito constructivo, y la participación de la mujer en estos procesos de aprendizaje.

8 cursos/talleres de construcción básica (sistemas y procesos constructivos de obra gruesa y fina), con especialidad en acabados - pintura decorativa- e instalaciones, eléctricas, sanitarias y de agua potable, los cuales han sido complementados con nociones básicas de lectura e interpretación de planos arquitectónicos, cálculo de volúmenes y áreas, presupuestos, supervisión, control de almacenes y supervisión de obra". (Red del Hábitat, 2012b, p. 50).

La estructura curricular de la capacitación se determinó a partir de módulos constructivos, a los que se les incorporaron aprendizajes sobre Equipos de Protección Personales (EPPs) y Salud Ocupacional, así como una etapa formativa, con propósitos de concientización sobre derechos humanos, de la mujer, laborales, a la ciudad y al hábitat. Luego, entonces, para la implementación de los módulos, se participó con facilitadores/as de diversas áreas del conocimiento, tanto constructivo como en humanidades y jurisprudencia. Por tanto:

Como es natural los primeros cursos sirvieron para evaluar y ajustar contenidos temáticos, cargas horarias, recursos pedagógicos y académicos a ser implementados en los cursos/taller. En este sentido, se debe destacar el trabajo interinstitucional desarrollado entre el Centro de Capacitación y Adiestramiento Municipal, CCAM del GAMLP y Red Hábitat, que de manera coordinada han elaborado la carpeta modular de enseñanza del curso/ taller, "Técnicas y Procedimientos para la Construcción de Obras en Infraestructura Urbana", que sirvió como base fundamental en el proceso de capacitación de las Mujeres Constructoras del Hábitat." (Red del Hábitat, 2012b, p. 30).

Por todo cuanto se ha localizado en relación a la capacitación de las Albañilas, se puede afirmar que enotros países latinoamericanos se han desarrollado programas de aprendizaje especializados en áreas relacionadas a la Albañilería, los acabados, la pintura, la plomería y la electricidad. Se trata de cursos y talleres que constituyen niveles completos de especialización (ver Figura 5). Luego, entonces, uno de los programas curriculares con mayor definición estructural resulta ser el que se diseñó en Argentina, impulsado por el Ministerio de Trabajo, Empleo y Seguridad Social. El incremento de la competitividad laboral del ramo constructivo, los cambios en la innovación tecnológica y el manejo de programas de calidad de las empresas empleadoras, han sido los factores impulsores de esta iniciativa. 


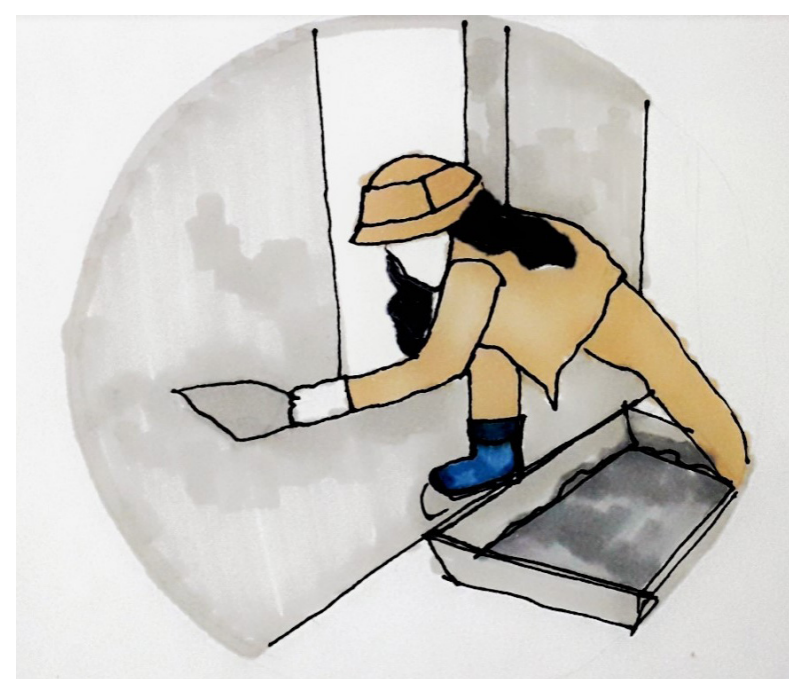

Figura 5. Programas de Aprendizaje Especializados Fuente: Flor Ríos Ventura.

El diseño curricular, está basado en competencias, organizado a partir de la agrupación de tareas constructivas por módulos y unidades de competencia. Así, a través de cuatro módulos, y cinco unidades de competencia, y la división de las etapas de aprendizaje, los saberes abordan temáticas relacionadas con la ejecución de cimientos y muros de elevación, la ejecución de estructuras sencillas de hormigón armado, la ejecución de terminaciones o superficies a revestir, y la formación sobre la gestión del proceso de trabajo. Por esa razón, las unidades de competencia se centraron en potenciar la organización de las actividades en la Albañilería tradicional, la construcción de cimientos y muros, la construcción de estructuras de hormigón armado, la construcción de terminaciones del proyecto y las condiciones de trabajo.

La planeación interna de cada uno de los módulos, está constituida por una estructura curricular que abarca una etapa introductoria, el desarrollo de objetivos de aprendizaje y sus competencias, el impulso de contenidos, las actividades prácticas, las actividades de cierre, los criterios de acreditación, y la bibliografía. Por tanto, se trata de un instrumento configurado con apoyo de profesionales de la educación, pues los módulos manifiestan una organización que responde a un aprendizaje por grado de complejidad. Entonces, el programa curricular:
Es la expresión del trabajo técnico, que hemos realizado en conjunto con los especialistas del área de la formación de diversos sectores de actividad, para establecer los parámetros de calidad de una oferta formativa que pretende satisfacer las demandas productivas y las necesidades formativas de los trabajadores y trabajadoras de nuestro país.

En términos de transparencia, estos documentos brindan información a las instituciones, los docentes, los empresarios y a quienes quieren formarse sobre los contenidos, la duración y los requerimientos de una oferta formativa reconocida sectorialmente. Por tal motivo, busca integrar contenidos conceptuales, procedimentales y actitudinales que favorezcan el acceso al empleo de calidad y que genere las competencias requeridas para la competitividad del sector y del país... (Sistema Nacional de Formación Continua y Certificación de Competencias, 2017, p. 38).

Ahora bien, resulta importante mencionar que esta propuesta curricular, formulada bajo sólidos criterios pedagógicos y profesionalizantes, representa, en comparación con otros de su misma especie, el instrumento de enseñanza-aprendizaje de mayor formalidad escolarizada. Solo que el documento carece de datos que referencien su aplicabilidad, y, por consecuencia, su proximidad y empatía entre la comunidad de las Albañilas.

\section{CONCLUSIONES}

De manera conclusiva puede advertirse que la integración de las mujeres como fuerza laboral en algunas de las áreas productivas, identificadas en otros tiempos como espacios exclusivos para varones, hoy resulta de gran interés dentro de los países latinoamericanos. El caso de las Albañilas está resultando ser un fenómeno laboral que ha experimentado un crecimiento, no solo en términos cuantitativos sobre su integración en obra, sino que ha incrementado las maneras de capacitación, lo que representa la sustitución de los aprendizajes empíricos hacia los aprendizajes escolarizados.

Las ofertas de cursos y talleres con participación de profesionales en construcción, en pedagogía, o en jurisprudencia, han posibilitado una formación 
más sólida para las mujeres constructoras, y, por lo tanto, mayores oportunidades de formalidad y seguridad laboral. Ciertamente, la escolarización de las mujeres Albañilas, les ha permitido adquirir capacidades que rebasan las actividades propias de la categoría del Albañil, pues llegan a dominar saberes relacionados con la pintura, la plomería, la electricidad o la decoración. Sin dejar de mencionar que algunas de ellas han demostrado tener la capacidad para ser operadoras de maquinaria pesada, o maestras de obra.

Pero, si bien es cierto que el ambiente laboral sigue siendo predominantemente desigual, hostil y riesgoso para la integridad de la mujer Albañil, resulta sorprendente conocer que las mujeres con formación universitaria en arquitectura, ingeniería y restauración, son quienes menos oportunidad laboral han brindado a las Albañilas. $Y$, aunque es probable que ello suceda por razones multifactoriales, no se puede dejar de señalar que las iniciativas existentes tan poco han dado amplios resultados.

Uno de los principales límites que han frenado la continuidad de los cursos y talleres ha sido el desinterés del Estado por impulsar políticas públicas que aseguren la permanencia formativa de las Albañilas, a lo que se le suma la poca credibilidad que los empleadores demuestran al evaluar en ellas los factores rendimiento y constancia laboral.

Luego, entonces, la investigación ha demostrado que resulta pertinente y emergente fortalecer las iniciativas de capacitación para las mujeres interesadas en la Albañilería. Asimismo, debe considerarse la integración de otros actores a los programas curriculares, como lo son los Albañiles(as) de mayor experiencia, que por su edad madura experimentan el desempleo. Se trataría de la formulación de equipos capacitadores, que estuviesen constituidos por profesionales de carrera, no solo en construcción, así como trabajadores retirados, de amplia experiencia en el oficio.
Se cree también que resulta imprescindible ampliar el alcance de estas capacitaciones, abarcando los ambientes rurales, puesto que cada mujer capacitada en las tareas de la Albañilería sería un elemento activo en el aprovechamiento de las mejoras de la arquitectura y urbanismo de la comunidad. Empero, también ello puede representar saberes estimuladores de integración comunitaria, pues ellas pueden formar comisiones de capacitación permanente, y desarrollar con ello una autoconstrucción de alta calidad y compatibilidad con los valores arquitectónicos de su identidad constructiva.

El incremento que en los últimos años ha experimentado el empoderamiento de la mujer profesional de la construcción, al constituirse en sociedades productivas y profesionalizantes, como el caso de las asociaciones de arquitectas, ingenieras, constructoras, etc., debe representar un medio desde el cual se impulsen las capacitaciones de las Albañilas y las oportunidades laborales, garantizando, con mayor empeño, la inclusión, la equidad, la seguridad y el crecimiento formativo.

Desde luego, que el presente artículo estuvo centrado de manera estratégica en el caso de las Albañilas; pero, no obstante, quedamos en deuda con aquellas mujeres que actualmente están siendo capacitadas a través de la arquitectura participativa, a las cuales no se les puede nombrar como Albañilas, puesto que no forzosamente se están capacitando en las tareas de la construcción para desarrollarse bajo los mismos fines. Se trata de una deuda que se pretende saldar a la brevedad, pues este primer acercamiento en relación con las mujeres menos visibles dentro de las tareas constructivas, resulta ser una invitación para emprender investigaciones desde diversos enfoques, alcances y propósitos. 


\section{REFERENCIAS}

Arias, P. (2016). El trabajo femenino: del permiso a la obligación. Papeles de población. 22(90):197-228. Recuperado de: https://www.redalyc.org/pdf/112/11249884008.pdf

González. E. (2017). G2 taller de arquitectura. Ayudante, Albañil y Maestro Albañil. [Mensaje en un blog] Recuperado de https://arquitecturag2.wordpress.com/

López B., M. T. (2010). El trabajo de las mujeres en el mundo urbano medieval. Mélanges de la Casa de Velázquez. 40-2 http://journals.openedition.org/mcv/3553

Marcos y Bausá, R. (1879). Manual del Albañil. España: Biblioteca Enciclopédica Popular llustrada. Recuperado de: https://issuu.com/anarchitect/docs/name386364

Mauro, A. (1986). Albañiles campesinos: migración temporal de los obreros de la construcción. Quito, Ecuador: CIUDAD, Centro de investigaciones. Recuperado de: https://biblio.flacsoandes.edu.ec/libros/5840-opac

RAE. (2019). Diccionario de la lengua española. España: Real Academia Española. Disponible en: https://dle. rae.es/alba\%C3\%B1il

Red del Hábitat (2012a). Identificación y fortalecimiento de agrupaciones de mujeres constructoras del Hábitat. En B. V., Diagnóstico socioeconómico de la mujer constructora (p. 8). Bolivia: Red Hábitat y Conexión Fondo de emancipación.

Red del Hábitat (2012b). Mujeres constructoras del hábitat. Bolivia: Taller de proyectos e investigación del hábitat urbano y rural Red hábitat.

Sánchez, G. (2014). Mi ambiente. Recuperado de http://www.miambiente.com.mx/comunitarias/aguasmaistros-mujeres-albaniles/

Sistema Nacional de Formación Continua y Certificación de Competencias. (2017). Formación basada en Competencias, Albañil en construcciones tradicionales. Argentina: Ministerio del trabajo, empleo y seguridad social. Recuperado de: http://www.trabajo.gov.ar/downloads/formacioncontinua/DC CONSTRUCCION Albanil en construcciones tradicionales.pdf

Universidad Nacional Autónoma de México. (2016). UNAM GLOBAL. Obtenido de http://www.unamglobal. unam. $m x / ? p=64147$

Universidad Veracruzana. (14 de Noviembre de 2017). 3er Foro regional de equidad y género, "Diferentes miradas". Obtenido de https://www.uv.mx/pozarica/foroequidad2017/files/2017/10/20.maria-erika-ariasbustos.pdf

Zambrana, H.; Saavedra, J. (1992). Proyecto Yacupaj. Manual de Albañilería para obras de saneamiento básico. Bolivia: Área técnica №5. Recuperado de: http://www.anesapa.org/wp-content/ uploads/2014/12/05MANAlbanileriaSanBas.pdf 